\title{
Analytic Approximations of SIR Compartmental Models of Infectious Dis- ease Epidemics
}

\section{Joel K Weltman}

Department of Medicine, Alpert/Brown University School of Medicine, Providence, RI, 02912 USA

\section{Keywords: Epidemiology; SIR; Sigmoid; Logistic}

A corner stone of the analysis of epidemics of infectious diseases is the SIR system [1] of Ordinary Differential Equations (ODEs). In SIR nomenclature, $\mathbf{S}$ is the number of Susceptible individuals, $\mathbf{I}$ is the number of Infected individuals and $\mathbf{R}$ is the number of Removed individuals, i.e., Removed either by recovery or by death. The SIR ODEs are solvable numerically $[2,3]$ but they do not have closed form solutions.

An approach to analysis of epidemics is proposed here which is conceptually based upon the SIR numerical solutions but which produces more easily interpreted, analytic results. This approach depends upon fitting, by regression, the summed count of infected individuals, $\mathrm{Y}$, to the expression:

$$
Y=\frac{c}{b+e^{-a t}}
$$

Where $\mathbf{a}, \mathbf{b}$ and $\mathbf{c}$ are parameters and $\mathrm{t}$ is time. In the instance of viral sequence counts from the 2009-2010 pH1N1 pandemic, the Pearson correlation coefficient ( $\mathbf{r}$ ) varied from a minimum of 0.9495 to a maximum of $0.9991, \mathbf{p}<2.22 \mathrm{e}-16$ at each of 23 geographic locations worldwide [4]. The first derivative of $\mathbf{Y}$ with respect to time (dY/dt ) is:

$$
\frac{d Y}{d t}=\frac{c a e^{-a t}}{\left(b+e^{-a t}\right)^{2}}
$$

$\mathrm{Y}$ and $\mathrm{dY} / \mathrm{dt}$ are assigned to compartments of the SIR system as follows: Y maps onto Susceptible(S) by subtraction from Total:

$$
\text { Total }-Y-d Y / d t->S
$$

Where Total equals the overall total count. dY/dt maps directly onto the number of infected (I):

$$
\mathrm{dY} / \mathrm{dt} \rightarrow \mathrm{I}
$$

and $\mathbf{Y}$ maps directly onto Removed (R ):

$$
Y \rightarrow R
$$

Plots of time-dependency of the functions, calculated according to the relationships $1-5$ above, with demonstration of the effects of variation of parameter a are shown in Figure 1. Such SIR-type functions can be modified with $\mathbf{t}-\mathbf{t}_{\mathbf{0}}$ time-shifts to allow for incubation time, duration of illness and other Effects as in SEIR [2] extensions of SIR.

The method proposed here is offered as an adjunct to SIR numerical solutions, an adjunct in which continuous, analytic functions with derivatives and anti derivatives (indefinite integrals) are produced. These functions, illustrated in Figure 1, calculated according to equations 1 and 2 and relationships $3-5$, above, may facilitate evaluation of epidemics and estimation of the evolutionary trajectory of infectious agents. Standardized curves of these functions may help development of earlywarning algorithmic systems for prediction of dangerous epidemics.

\section{References}

1. Kermack WO, McKendrick AG (1927) A Contribution to the Mathematical Theory of Epidemics. Proc R Soc Lond A 115: 700-721.

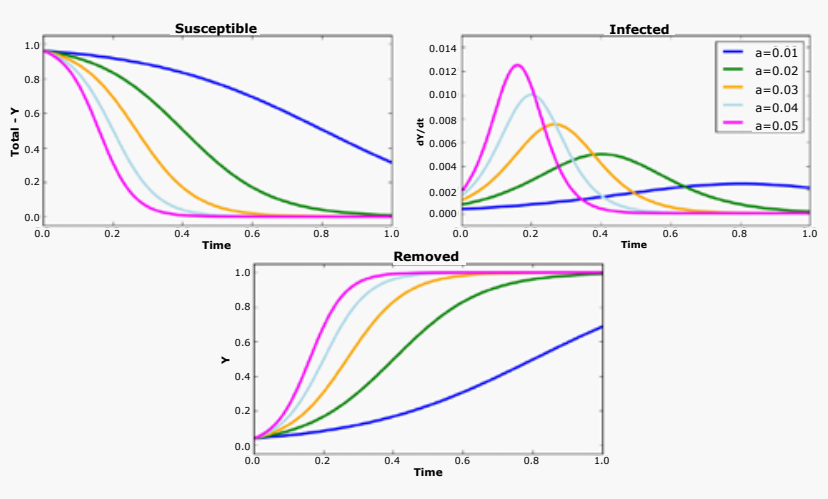

Figure 1: SIR Analysis of a Hypothetical Epidemic Approximated by $Y$ and $d Y / d t$. (top left ) Susceptible (S) approximated by Total - Y - dY/dt, (top right) Infected (I) approximated by $\mathbf{d Y} / \mathbf{d t}$ and (bottom) Removed (R) approximated by by $\mathbf{Y}-$ dY/dt. The non-linear parameter a was varied from 0.01 to 0.05 .

2. Keeling MJ, Rohani $P$ (2007) Modeling Infectious Diseases in Humans and Animals. Princeton University Press Princeton NJ USA.

3. Radhakrishnan K, Hindmarsh AC (1992) Description and Use of LSODE, the Livermore Solver for Ordinary Differential Equations. Technical report UCRLID-113855, Lawrence Livermore National Laboratory, December 1992.

4. Thompson WA, Weltman JK (2012) Bioinformatic Analyses of 2009-2010 Pandemic H1N1 Influenza A Hemagglutinin Subsets. J Med Microbiol Diagn.

${ }^{*}$ Corresponding author: Joel K Weltman, Department of Medicine, Alpert/Brown University School of Medicine, Providence, RI, 02912 USA, E-mail: joel_weltman@brown.edu

Received June 19, 2012; Accepted June 20, 2012; Published June 25, 2012

Citation: Weltman JK (2012) Analytic Approximations of SIR Compartmental Models of Infectious Disease Epidemics. J Med Microb Diagn 1:e110. doi:10.4172/2161 0703.1000e110

Copyright: (c) 2012 Weltman JK. This is an open-access article distributed under the terms of the Creative Commons Attribution License, which permits unrestricted use, distribution, and reproduction in any medium, provided the original author and source are credited. 